\title{
Predictors and nomogram models for postoperative headache in patients undergoing heart valve surgery
}

\author{
Dashuai Wang, Xiaofan Huang, Hongfei Wang, Sheng Le, Xinling Du \\ Department of Cardiovascular Surgery, Union Hospital, Tongji Medical College, Huazhong University of Science and Technology, Wuhan, China \\ Contributions: (I) Conception and design: X Du, X Huang, D Wang; (II) Administrative support: X Du, H Wang, S Le; (III) Provision of study \\ materials or patients: X Du, X Huang, H Wang; (IV) Collection and assembly of data: D Wang, S Le, H Wang; (V) Data analysis and interpretation: \\ D Wang, S Le, X Huang; (VI) Manuscript writing: All authors; (VII) Final approval of manuscript: All authors. \\ Correspondence to: Xiaofan Huang, MD. Union Hospital, Wuhan Jiefang Road, No. 1277, Wuhan 430022, China. Email: Dr_xfhuang@hust.edu.cn; \\ Xinling Du, MD, PhD. Union Hospital, Wuhan Jiefang Road, No. 1277, Wuhan 430022, China. Email: xinlingdu@hust.edu.cn.
}

Background: Headache is a frequent complication after cardiac surgery. However, studies on the risk factors of postoperative headache $(\mathrm{POH})$ are rare. The purpose of this study was to identify independent risk factors for $\mathrm{POH}$ in patients undergoing heart valve surgery (HVS) and to develop and validate risk prediction models.

Methods: Consecutive patients undergoing open HVS from 2016 to 2019 were enrolled in this study. Patients were randomly assigned to training and validation sets at a 2:1 ratio. Univariate and multivariate analysis were applied to identify independent predictors for $\mathrm{POH}$ in the training set. A nomogram predicting $\mathrm{POH}$ was developed based on these factors, and was validated in the independent validation set.

Results: POH developed in 1,061 of the 3,853 patients $(27.5 \%)$. The overall mortality was $2.9 \%$, and it was significantly higher in patients with $\mathrm{POH}$ (4.3\% versus $2.4 \%, \mathrm{P}<0.001)$. In the training set, six independent predictors were identified by multivariate analysis, including female, smoking history, hypertension, headache history, left ventricular ejection fraction, and cardiopulmonary bypass time. The model demonstrated good discrimination in both the training (c-index: 0.811) and validation sets (c-index: 0.814), and calibration was assessed by visual inspection. A second nomogram was also constructed including only preoperative predictors, with good discrimination (c-index: 0.792) and calibration. The decision and clinical impact curves of the models showed good clinical utility.

Conclusions: We developed and validated two risk prediction models for $\mathrm{POH}$ in patients undergoing HVS. The models may have clinical utility in individualized risk assessment and preventive interventions.

Keywords: Headache; heart valve surgery (HVS); risk factor; prediction model; nomogram

Submitted Apr 12, 2021. Accepted for publication Jun 04, 2021.

doi: $10.21037 /$ jtd-21-644

View this article at: https://dx.doi.org/10.21037/jtd-21-644

\section{Introduction}

Cardiovascular disease remains the leading cause of mortality and disease burden in the world $(1,2)$ The prevalence and related health care costs of cardiovascular disease have been rising steadily these years (1). Valvular heart disease is one of the most common cardiovascular diseases and surgical intervention is the main treatment for severe valvular heart disease (3). Clinically, it has been observed that a considerable proportion of patients develop headaches after heart valve surgery (HVS).

Postoperative headache $(\mathrm{POH})$ is a common complication after surgical procedures, associated with increased morbidity, prolonged hospital stay, reduced quality of life, and additional economic burden (4-10). The incidence of $\mathrm{POH}$ reported in the literature varies substantially after different types of surgery (4,6,9,11-17). Currently, some studies have been carried out to identify possible predictors 
for the occurrence of $\mathrm{POH}$ in patients undergoing craniotomy, general, spinal, orthopedic, gynecologic, and ear-nose-throat procedures $(6,13,16,18-21)$. Several related risk factors have been reported, such as age, female gender, previous history of headache, and anaesthetic drugs $(13,18,22)$. However, there are only few reports available about the development of $\mathrm{POH}$ after cardiac surgery, and basically it's just used as a secondary outcome (23). To our knowledge, there are no prior studies identifying the risk factors of $\mathrm{POH}$ after cardiac surgery, let alone a risk model that can be used to early predict the occurrence of $\mathrm{POH}$. The establishment of a convincing prediction model for $\mathrm{POH}$ after cardiac surgery is in urgent need and we therefore conducted this prospective observational study.

The purpose of this study was to identify independent risk factors for the development of $\mathrm{POH}$ in adult patients who underwent open HVS, and to develop as well as validate an easy-to-use clinical prediction model that may help clinicians assess the risk of $\mathrm{POH}$ early. We present the following article in accordance with the TRIPOD reporting checklist (available at https://dx.doi.org/10.21037/jtd-21-644).

\section{Methods}

The study was conducted according to the Declaration of Helsinki (as revised in 2013). The Ethics Committee of Tongji Medical College of Huazhong University of Science and Technology (IORG No. IORG0003571) approved this study. The need for informed consent was waived because of the retrospective study design.

\section{Study population}

Consecutive adult patients (age $\geq 18$ years) who underwent open HVS in our hospital between 2016 and 2019 were included in this study. Exclusion criteria were intraoperative or early postoperative death, unconsciousness after surgery, and records with missing data. The remaining patients were divided into two groups according to the presence or absence of any kind of headache from the first postoperative day to discharge.

\section{Data collection and variables}

Clinical data were collected through the hospital's electronic medical record management. Pre-, intra-, and post-operative variables were analyzed. Demographics included sex, age, body mass index, smoking history and drinking history. Comorbidities included hypertension, diabetes mellitus, chronic obstructive pulmonary disease, gastrointestinal tract disease, peripheral vascular disease, cerebrovascular disease, renal insufficiency, atrial fibrillation, pulmonary artery hypertension, left ventricular ejection fraction (LVEF), pericardial effusion, headache history, general surgical history, and cardiac surgery history. Laboratory values included white blood cell (WBC) count, red blood cell count, hemoglobin, platelet count, serum creatinine, albumin, and globulin. Intraoperative variables included transfusion of red blood cells, the duration of cardiopulmonary bypass (CPB) and aortic cross clamp. Postoperative variables included pneumonia, reintubation, tracheotomy, readmission to intensive care unit (ICU), mortality, the lengths of mechanical ventilation, ICU and hospital stay.

\section{Definitions of variables}

Body mass index was calculated as weight $(\mathrm{kg})$ divided by height squared. Smoking history was positive if there was current or previous daily smoking. Hypertension referred to blood pressure $\geq 140 / 90 \mathrm{mmHg}$, use of anti-hypertensive drugs, or previous diagnosis of hypertension. Chronic obstructive pulmonary disease was defined as FEV1/FVC $\leq 0.7$. Diabetes mellitus was defined as fasting glucose $\geq 7.0 \mathrm{mmol} / \mathrm{L}$, random glucose $\geq 11.1 \mathrm{mmol} / \mathrm{L}$, use of diabetic medication, or previous diagnosis of diabetes mellitus. Headache history was defined as any report of either migraine or any other recurrent kind of headache in referral letters or hospital medical records. Renal insufficiency referred to serum creatinine level higher than $110 \mu \mathrm{mol} / \mathrm{L}$ or previous diagnosis of renal insufficiency.

\section{Statistical analysis}

We randomly divided the overall dataset into the training set and the validation set, with a ratio of $2: 1$. The former was used for the development of the model, whereas the latter was used for the validation of the model. In the training set, univariate analysis of preoperative and intraoperative variables was used to screen possible risk factors. Normally distributed continuous variables were presented as mean \pm standard deviation and analyzed by two-independent samples $t$ test. Non-normally distributed continuous variables were expressed as median with inter-quartile range and compared by Mann-Whitney $\mathrm{U}$ 


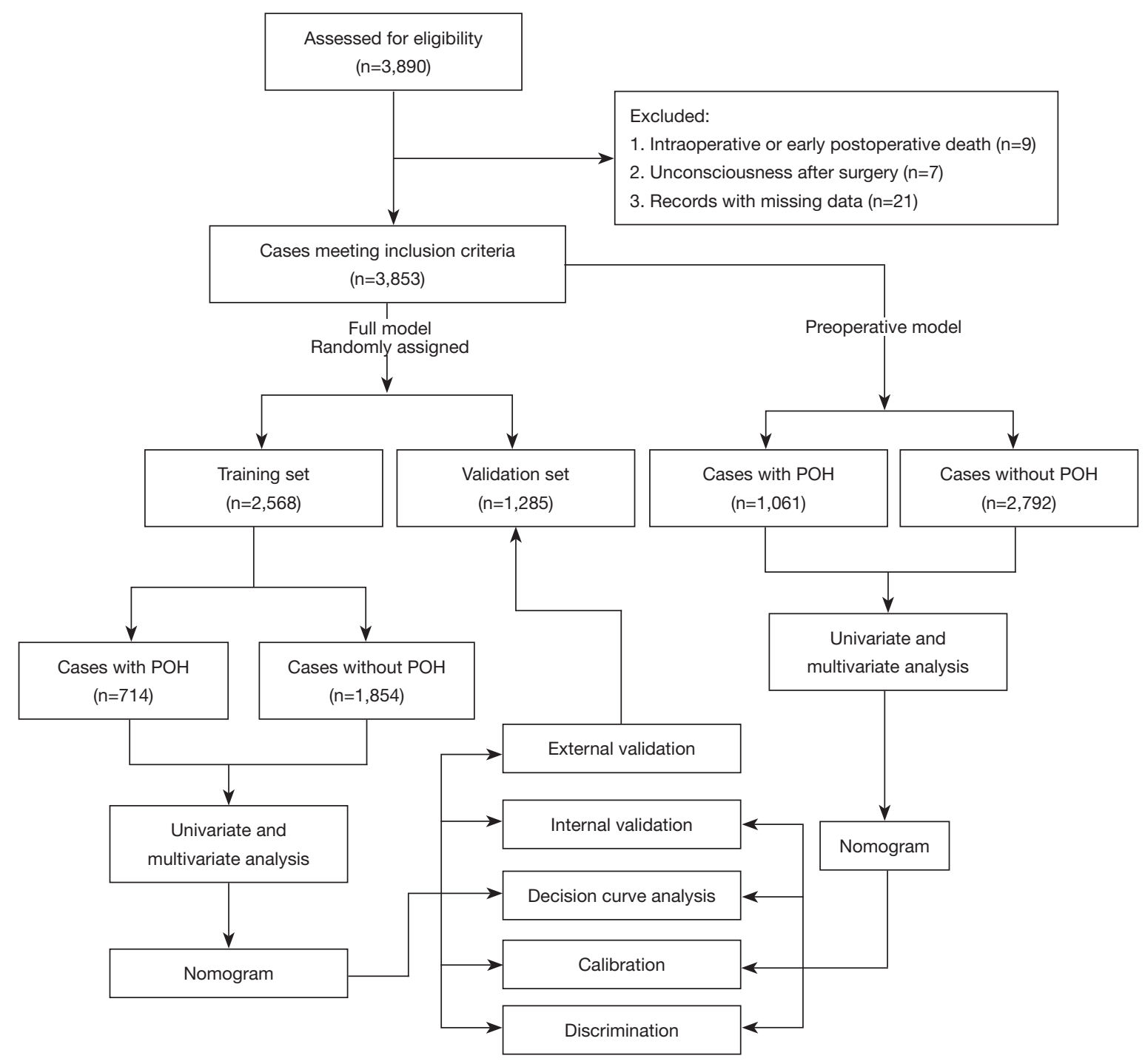

Figure 1 Flow chart of the study. POH, postoperative headache.

test. Categorical variables were expressed as frequency (percentage) and analyzed by the chi-square test or Fisher's exact test. Variables screened in the univariate analysis were then entered into the multivariate analysis to identify independent risk factors. A nomogram based on the full multivariate logistic regression model was constructed to predict the probability of $\mathrm{POH}$ after HVS. In the same way, a second multivariate nomogram model that only included preoperative variables was developed using the overall dataset.

Internal validation of the prediction model was evaluated by bootstrapping using 1,000 resamples. External validation was conducted in the independent validation set. Calibration curves were plotted to visually assess the calibration. Discrimination was assessed using the area under the receiver operating characteristic (ROC) curve (AUC) or c-index. Comparison of the AUC between the training and validation sets was performed using the Delong method (24). Decision curve analysis was applied to evaluate the clinical utility of the model (25). The decision curve displayed the standardized net benefit against the threshold probability. The clinical impact curve showed the number of estimated high risk with different threshold probability and the number of true positives among 1,000 patients. A study flowchart is provided in Figure 1.

Two-tailed $\mathrm{P}$ values less than 0.05 were considered statistically significant. Statistical analyses were performed using R software (version 4.0.3). 


\section{Results}

\section{Demographic characteristic}

Among the 3,890 patients undergoing HVS, nine died intraoperatively or early postoperatively, seven lapsed into unconsciousness after surgery, and twenty-one had missing data. The remaining 3,853 cases fulfilled the inclusion criteria and were further analyzed (Figure 1). The average age of the included patients was $51.27 \pm 12.56$ years; $53.9 \%$ were men. The overall incidence of $\mathrm{POH}$ after HVS was $27.5 \%$.

All patients underwent sternotomy and cardiopulmonary bypass, in which $59.8 \%$ underwent single-valve surgery. Approximately $63.6 \%$ of patients underwent mitral valve surgery and more than half underwent aortic valve surgery. About two-thirds of mitral valve surgery and a quarter of aortic valve surgery were repair procedures. In patients undergoing valve replacement surgery, about twofifths used biological prosthesis. Regarding the valvular pathologies, stenosis accounted for about one-third of mitral valve diseases and about one-fifth of aortic valve diseases.

The patients constituting this study population had different comorbidities. Patients with smoking history made up $26.8 \%$, drinking history $20.1 \%$, headache history $10.8 \%$, hypertension $24.3 \%$, diabetes mellitus $5.7 \%$, chronic obstructive pulmonary disease $12.8 \%$, gastrointestinal tract disease $8.2 \%$, renal insufficiency $8.3 \%$, atrial fibrillation $23.1 \%$, cardiac surgery history $8.0 \%$. The average volume of intraoperative transfusion of red blood cells was 4.0 $(3.0,7.5)$ units, and the average CPB duration was 108 (86, 139) minutes. The baseline characteristics, comorbidities and operative variables were similar for the training and validation sets (Table 1). The incidence rate of $\mathrm{POH}$ in the two sets was respectively $27.8 \%$ and $27.0 \%(\mathrm{P}=0.60)$.

Short-acting analgesics were used for postoperative pain control in our hospital. In this study, about $17.6 \%$ of patients had taken painkillers at least once for wound pain or other causes before the development of $\mathrm{POH}$ and the incidence of $\mathrm{POH}$ in these patients was $28.7 \%$. The other $82.4 \%$ of patients had not taken pain medication before the development of $\mathrm{POH}$ and the corresponding incidence was $27.3 \%$. No significant difference was found between groups $(\mathrm{P}=0.473)$, indicating that the use of painkillers may not have much effect on the detection rate of $\mathrm{POH}$.

\section{Development of the full nomogram model}

We initially conducted univariate analysis to screen possible risk factors for $\mathrm{POH}$ after HVS in the training set (Table 2). Collinearity diagnostics were performed before the construction of a multivariate model. Significant predictors in the univariate analysis were then entered into the multivariate analysis. By stepwise forward selection, we identified six independent risk factors in the final multivariate logistic regression model, including female, smoking history, hypertension, headache history, LVEF, and CPB time (Table 3). A nomogram predicting the probability of POH after HVS was then established on the basis of the full multivariate model (Figure 2). Regression coefficients of the predictors were scaled to points of $0-100$, which can reflect their relative importance.

The risk of $\mathrm{POH}$ after HVS in a specific patient can be easily predicted by summing the points of all variables. Female patients who had smoking history, hypertension, headache history, low LVEF and longer CPB time would have higher points and thus higher risk of $\mathrm{POH}$ after HVS. The risk of $\mathrm{POH}$ after HVS predicted by this nomogram model ranged from 0.01 to 0.99 .

\section{Assessment and validation of the full model}

The nomogram model was validated using both internal and external validation. Internal validation was performed via a bootstrap method with 1,000 resamples in the training set. External validation was conducted in the independent validation set. The goodness-of-fit of the model was tested by calibration plot. By visual inspection, the nomogram was well calibrated in both the training and validation sets (Figure 3). The ROC curves were drawn to evaluate the effectiveness of the nomogram model at predicting the probability of $\mathrm{POH}$ in patients undergoing HVS (Figure 4). The AUC was respectively 0.811 (95\% CI, 0.792-0.831) and 0.814 (95\% CI, 0.787-0.841) in the training and validation sets, demonstrating good discrimination. No significant difference was found between the two AUCs $(\mathrm{P}=0.90)$.

\section{Preoperative prediction model}

The full nomogram model above was constructed using both preoperative and intraoperative predictors. To facilitate clinical applications, we constructed a second model using only preoperative variables. By univariate and multivariate analysis, we identified seven preoperative independent predictors, including female, smoking history, hypertension, headache history, LVEF, WBC, and serum albumin (Table 4). A preoperative nomogram model was then constructed 
Table 1 Comparison of characteristics between the training and validation sets

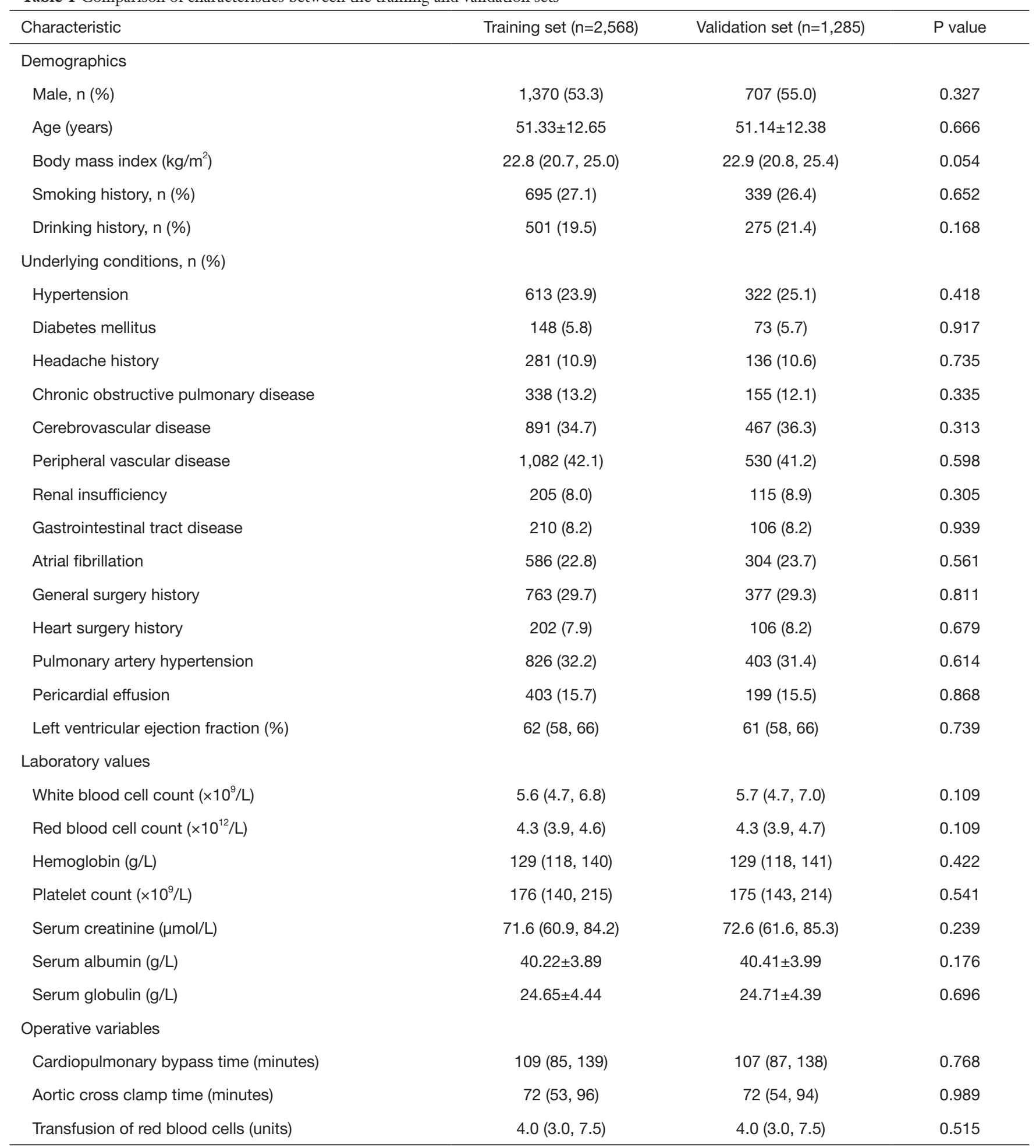


Table 2 Univariate analysis of possible risk factors for POH after HVS in the training set

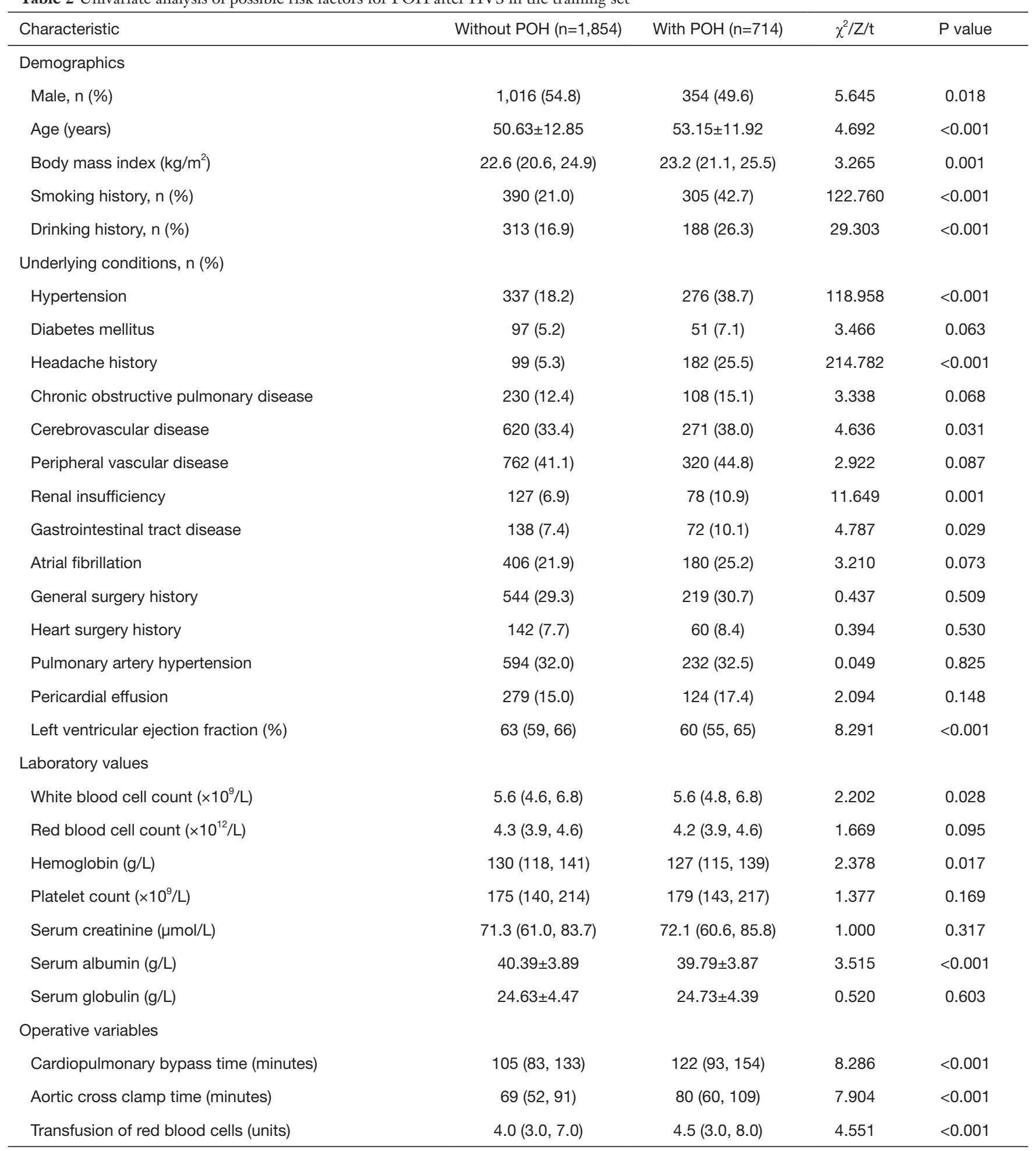

$\mathrm{POH}$, postoperative headache, HVS, heart valve surgery. 
Table 3 Multivariate analysis of independent risk factors for POH after HVS

\begin{tabular}{|c|c|c|c|c|}
\hline Characteristic & Coefficient & Standard error & OR $(95 \% \mathrm{Cl})$ & $P$ value \\
\hline Smoking history & 2.126 & 0.152 & $8.378(6.223-11.279)$ & $<0.001$ \\
\hline Hypertension & 1.295 & 0.117 & 3.651 (2.903-4.594) & $<0.001$ \\
\hline Headache history & 2.302 & 0.156 & 9.997 (7.366-13.567) & $<0.001$ \\
\hline CPB time (minutes) & 0.009 & 0.001 & $1.009(1.007-1.011)$ & $<0.001$ \\
\hline Intercept & -2.313 & 0.482 & 0.099 & $<0.001$ \\
\hline
\end{tabular}

$\mathrm{POH}$, postoperative headache; HVS, heart valve surgery; Cl, confidence interval; CPB, cardiopulmonary bypass; LVEF, left ventricular ejection fraction; OR, odds ratio.

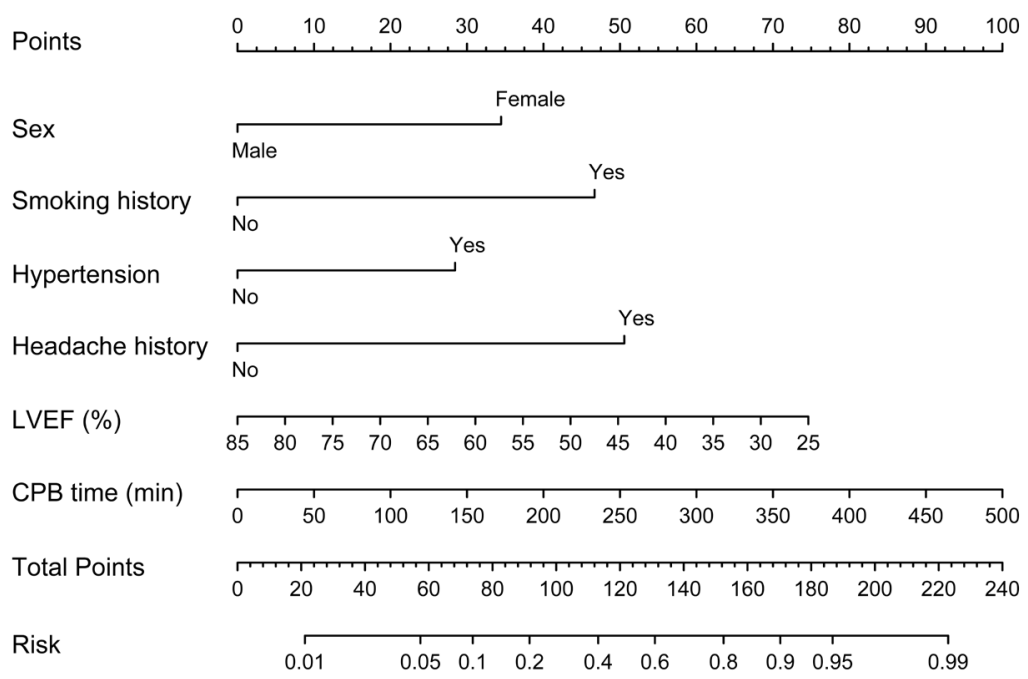

Figure 2 Nomogram for the prediction of postoperative headache in patients undergoing heart valve surgery. LVEF, left ventricular ejection fraction; $\mathrm{CPB}$, cardiopulmonary bypass.

based on the multivariate analysis (Figure $5 A$ ). The model was developed using data from all the 3,853 patients, and was validated using only internal validation with 1,000 bootstrap resamples. The calibration was good by visual inspection (Figure 5B). The AUC was 0.792 (95\% CI, 0.775-0.808), indicating reasonable discrimination (Figure 5C).

\section{Clinical utility of the two models}

To assess the clinical utility of the risk prediction models, decision curve analyses were performed along with graphical decision curves and clinical impact curves. The decision curves of both the preoperative model and the full model in the training and validation sets demonstrated that compared with "intervention for all" or "no intervention" strategies, our risk prediction models could obtain more clinical net benefits when the risk threshold was between 0.05 and 0.80 (Figure 6A). The clinical impact curves also illustrated that both models had remarkable predictive power and exhibited good clinical utility (Figure 6B,C,D).

\section{Outcome}

POH developed in 1,061 of the 3,853 patients $(27.5 \%)$. The overall mortality rate was $2.9 \%(112 / 3,853)$, with a rate of 

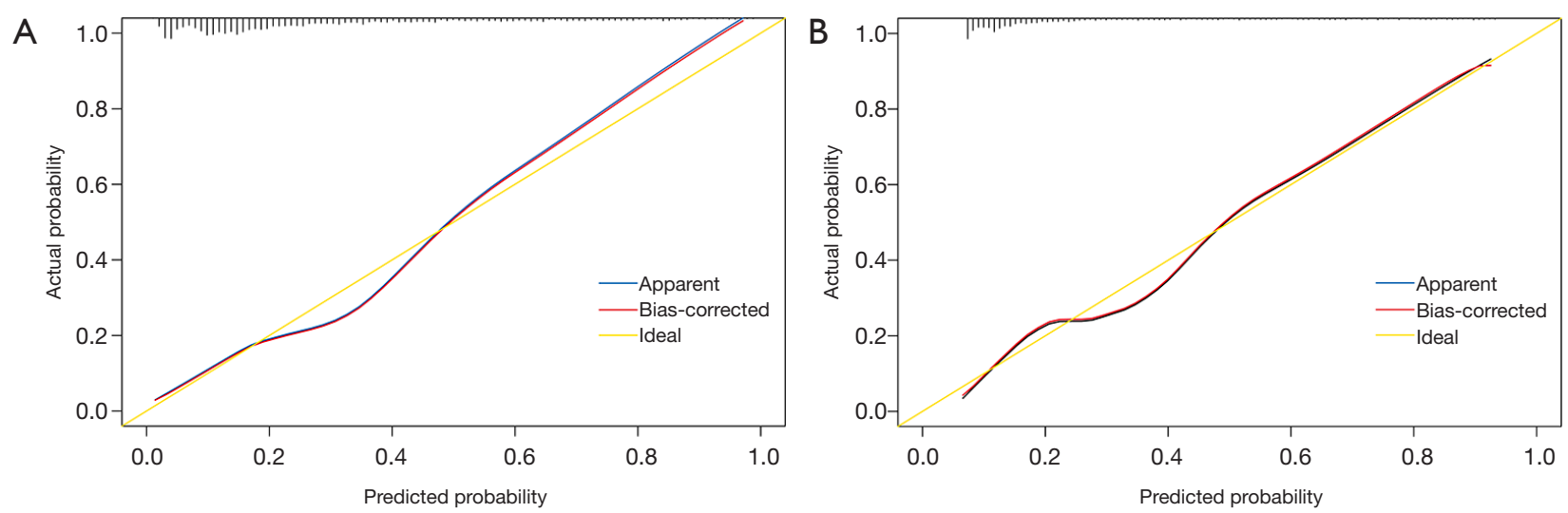

Figure 3 Calibration plots of the nomogram for the probability of postoperative headache after heart valve surgery in the training set (A) and the validation set.

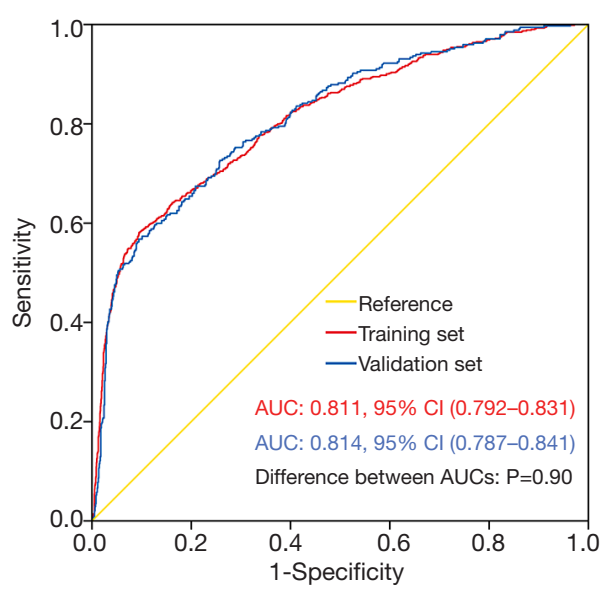

Figure 4 ROC curves for the full nomogram model in the training and validation sets. The AUC was 0.811 (95\% CI, 0.792-0.831) in the training set and 0.814 (95\% CI, 0.787-0.841) in the validation set. The difference between the two AUCs was not significant $(\mathrm{P}=0.90)$. AUC, area under the receiver operating characteristic curve; CI, confidence interval; ROC, receiver operating characteristic.

$4.3 \%$ in patients with $\mathrm{POH}$ versus $2.4 \%$ in those without $\mathrm{POH}$ [odds ratio $(\mathrm{OR})=1.872, \mathrm{P}=0.001]$. In addition, $\mathrm{POH}$ was associated with a significantly higher rate of postoperative pneumonia, reintubation and tracheotomy. The postoperative duration of mechanical ventilation, ICU and hospital stay also extended significantly in patients with $\mathrm{POH}$. Comparisons of postoperative variables between patients with and without $\mathrm{POH}$ after HVS are presented in Table 5.

\section{Discussion}

$\mathrm{POH}$ has been considered related to increased morbidity and mortality (4-8), which was reaffirmed by the results of our study. The incidence of $\mathrm{POH}$ after HVS was $27.5 \%$. The overall mortality rate was $2.9 \%$. However, the mortality rate in patients with $\mathrm{POH}$ was significantly higher than that in patients without $\mathrm{POH}$. In addition, significantly higher rates of postoperative pneumonia, reintubation and tracheotomy, and longer postoperative durations of mechanical ventilation, ICU and hospital stay were observed in patients with $\mathrm{POH}$. The higher morbidity and mortality in patients with $\mathrm{POH}$ emphasized the need of identifying independent risk factors and developing prediction models.

There have been several studies that looked at the risk factors for $\mathrm{POH}$ after surgical procedures in other diseases $(6,13,18,19,21)$. However, studies conducted in patients undergoing cardiac surgery are still lacking in this field. To our knowledge, the work that we report represents the first clinical prediction model for $\mathrm{POH}$ after HVS.

In this study, we used data from 3,853 patients who underwent HVS at our institution to develop and validate a full prediction model and a preoperative model for $\mathrm{POH}$. In the full model, six independent predictors associated with the development of $\mathrm{POH}$ were identified by multivariate logistic regression analysis, including female, smoking history, hypertension, headache history, LVEF, and CPB time. In the preoperative model, in addition to the five preoperative variables identified above, $\mathrm{WBC}$ and albumin were also identified as independent predictors. Nomograms based on the multivariate logistic regression models were 
Table 4 Multivariate analysis of independent preoperative risk factors for POH after HVS

\begin{tabular}{|c|c|c|c|c|}
\hline Characteristic & Coefficient & Standard error & OR $(95 \% \mathrm{Cl})$ & $P$ value \\
\hline Smoking history & 2.094 & 0.122 & $8.113(6.382-10.315)$ & $<0.001$ \\
\hline Hypertension & 1.276 & 0.092 & 3.581 (2.988-4.292) & $<0.001$ \\
\hline Headache history & 2.156 & 0.124 & $8.640(6.775-11.019)$ & $<0.001$ \\
\hline WBC $\left(\times 10^{9} / \mathrm{L}\right)$ & 0.040 & 0.019 & $1.041(1.003-1.081)$ & 0.033 \\
\hline Albumin $(g / L)$ & -0.030 & 0.011 & $0.970(0.950-0.991)$ & 0.005 \\
\hline Intercept & -0.480 & 0.564 & 0.619 & 0.394 \\
\hline
\end{tabular}

$\mathrm{POH}$, postoperative headache; HVS, heart valve surgery; $\mathrm{Cl}$, confidence interval; OR, odds ratio; LVEF, left ventricular ejection fraction; WBC, white blood cell.

A

Points

Sex

Smoking history

Hypertension

Headache history

LVEF (\%)

Albumin (g/L)

WBC $\left(\times 10^{9} / \mathrm{L}\right)$

Total Points

Risk
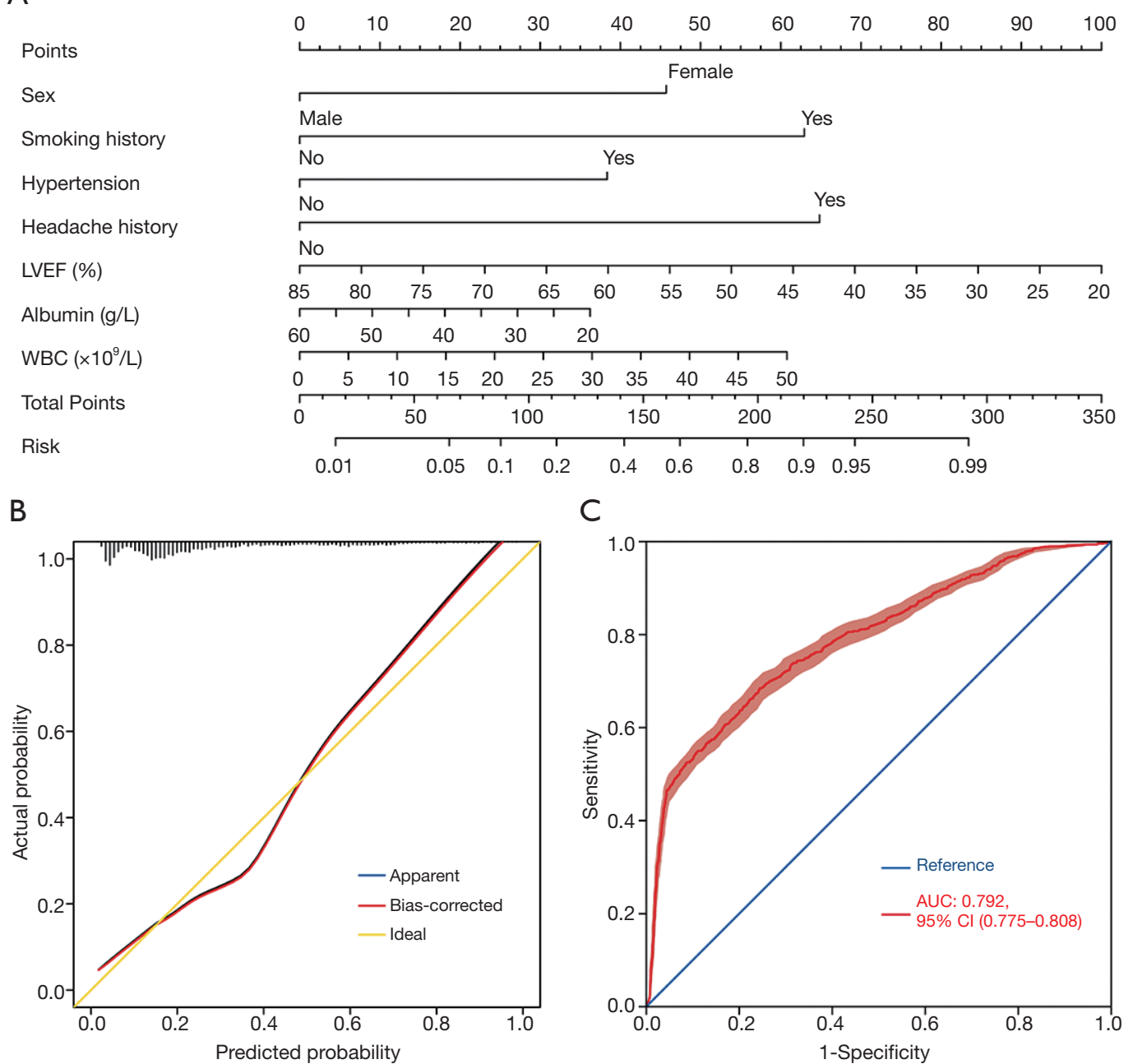

C

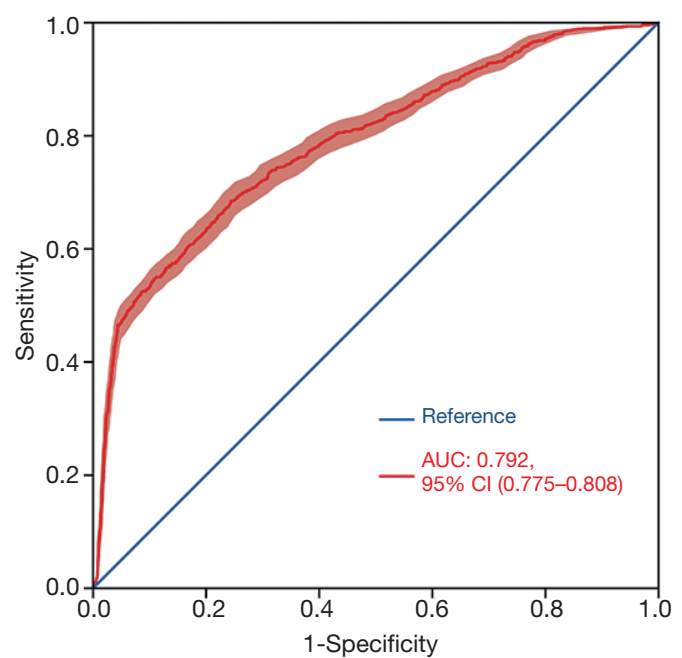

Figure 5 Nomogram of the preoperative prediction model (A) and corresponding calibration plot (B) and ROC curve (C). Good calibration and discrimination can be observed. LVEF, left ventricular ejection fraction; WBC, white blood cell. 
A

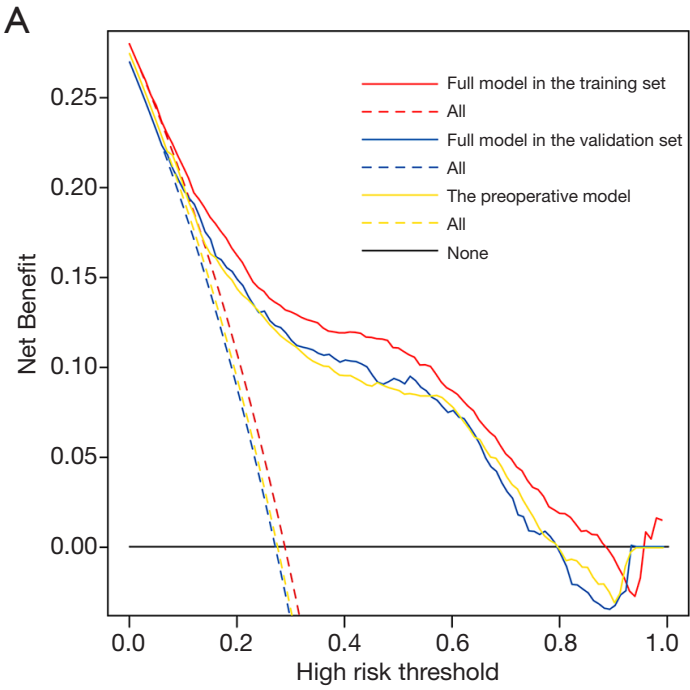

C

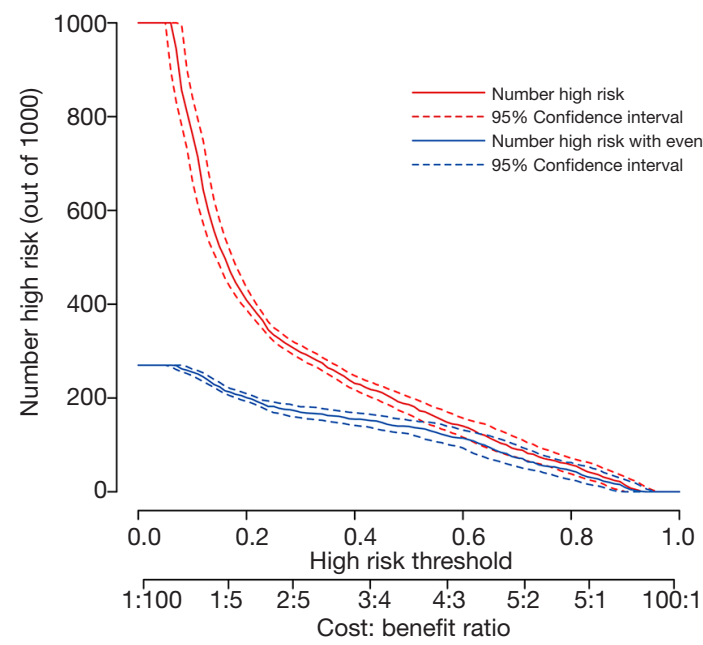

B

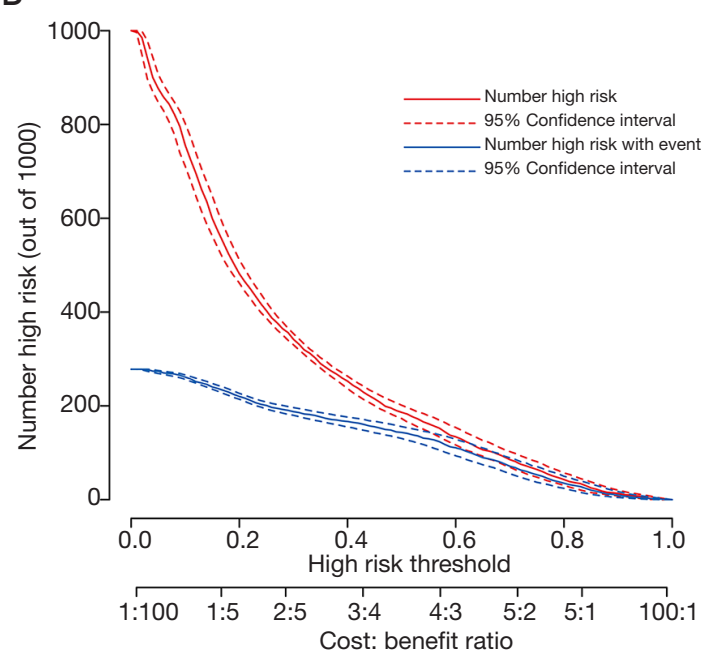

D

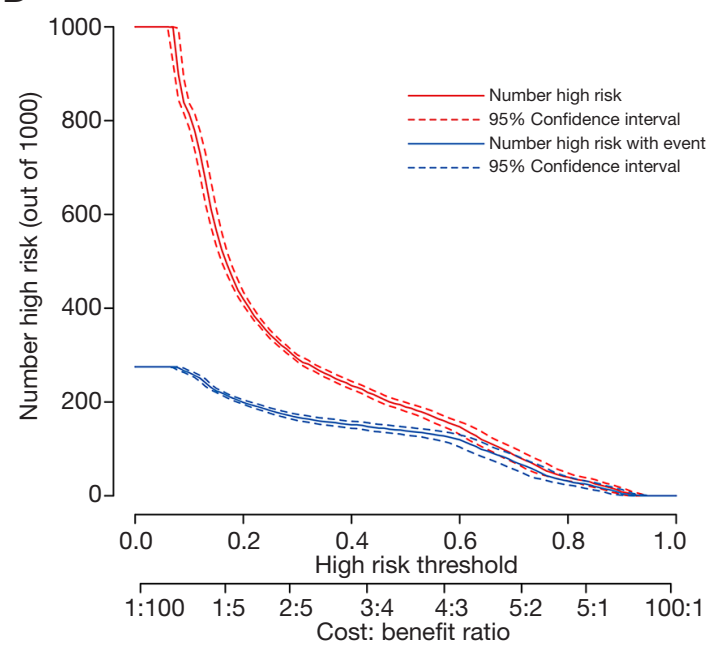

Figure 6 Decision curve analysis of both the preoperative model and the full model in the training and validation sets. (A) Decision curves of the full model in the training set (red line) and the validation set (blue line), and the preoperative model (yellow line) are shown. The "All" lines assume all patients are at high risk of $\mathrm{POH}$ and therefore prevent or treat everyone. The "None" line assumes all patients are at low risk of $\mathrm{POH}$ and therefore prevent or treat no one. The vertical axis displays net benefit and the horizontal axis shows the threshold probability. Clinical impact curves of the full model in the training set (B) and the validation set (C), and the preoperative model (D) are shown. Of 1,000 patients, the red lines show the total number that would be deemed high risk for each risk threshold. The blue lines show how many of those would be true positives (cases). The two horizontal axes show the correspondence between risk threshold and cost:benefit ratio. POH, postoperative headache.

then constructed. Both nomogram models showed good discrimination and calibration. The decision and clinical impact curves of the nomogram models also indicated remarkable predictive power and good clinical utility.

Although significant predictors for $\mathrm{POH}$ after surgery vary considerably in different reports, female gender as an independent risk factor for $\mathrm{POH}$ has been widely identified in the literature $(10,13,18,20,26,27)$, which was reconfirmed by our results. Paraskevi and colleagues reported that compared with male patients, the risk of $\mathrm{POH}$ increased significantly in female patients $(\mathrm{OR}=2.1, \mathrm{P}=0.024)$ (13). They believed that hormones and psychological factors may be responsible for the sex difference (28-30). Myles and colleagues found that females were 2.3 times more 
Table 5 Postoperative variables in patients with and without $\mathrm{POH}$ after HVS

\begin{tabular}{|c|c|c|c|c|c|}
\hline Variables & All patients $(n=3,853)$ & Without $\mathrm{POH}(\mathrm{n}=2,792)$ & With $\mathrm{POH}(\mathrm{n}=1,061)$ & $\chi^{2} / Z$ & $P$ value \\
\hline Reintubation & $157(4.1)$ & $101(3.6)$ & $56(5.3)$ & 5.424 & 0.020 \\
\hline Tracheotomy & $71(1.8)$ & $38(1.4)$ & $33(3.1)$ & 13.006 & $<0.001$ \\
\hline Mechanical ventilation (h) & $22(19,42)$ & $22(18,40)$ & $25(20,46)$ & 7.570 & $<0.001$ \\
\hline ICU stay (days) & $3(2,5)$ & $3(2,4)$ & $3(2,5)$ & 8.327 & $<0.001$ \\
\hline Hospital stay (days) & $14(11,19)$ & $14(11,18)$ & $15(11,20)$ & 5.089 & $<0.001$ \\
\hline Mortality & $112(2.9)$ & $66(2.4)$ & $46(4.3)$ & 10.590 & 0.001 \\
\hline
\end{tabular}

$\mathrm{POH}$, postoperative headache; HVS, heart valve surgery; ICU, intensive care unit.

likely to report $\mathrm{POH}$ (27). They hypothesized that males experienced a similar rate of $\mathrm{POH}$ in fact but underreported them. Expression of emotions such as pain or headache was more socially acceptable for women and thus they were more willing to describe them. However, males were often encouraged not to express such emotions and they were more likely to neglect these problems (27). In addition, studies have shown that female patients were more likely to find $\mathrm{POH}$ disabling and be adversely affected than male patients $(8,10)$.

Hypertension was also identified as a significant predictor for $\mathrm{POH}$ in our analysis, consistent with previous reports. Pamela and colleagues reported that hypertension was closely related to migraine headache, and some antihypertensive drugs may have beneficial effects on the frequency and severity of the headache attacks $(31,32)$. Beta-blockers and calcium channel blockers have been strongly recommended for the prophylaxis of $\mathrm{POH}$ in patients with hypertension $(33,34)$. Recently, the reninangiotensin system, which plays an important role in blood pressure regulation, has been reported to be involved in the pathogenesis of headache through susceptibility to oxidative stress, neurogenic inflammation, endothelial dysfunction, and neuromodulation of nociceptive transmission. Angiotensin converting enzyme inhibitors and angiotensin receptor blockers can also play a major role in the prophylactic management of headache (35). What's more, a compelling genetic correlation between blood pressure and migraine headache was identified through a genetic correlation analysis using cross-trait linkage disequilibrium score regression, suggesting that neurovascular processes related to increased blood pressure may underlie headache (36).
Smoking history was another independent risk factor for POH after HVS in this study. This was consistent with previous reports (13). Paraskevi and colleagues found that smoking was independently associated with $\mathrm{POH}$ (OR $=1.74, \mathrm{P}=0.006$ ). Although the specific mechanism remains unclear, we speculate that it may be related to tobacco withdrawal or vascular changes caused by smoking. Thus, it should be strongly recommended to stop smoking both in patients with high risk and in general population for general health maintenance and long-term primary prevention.

Headache history was the most important predictor identified in our analysis for the development of $\mathrm{POH}$ after HVS. This was consistent with the results reported in the literature $(10,21,37)$. Valentinis and colleagues reported that a longstanding headache history was the only significant independent intrapersonal risk factor for the development of $\mathrm{POH}(\mathrm{OR}=3.07, \mathrm{P}=0.01)$ (37). Yabuki and colleagues reported that patients with preoperative headache had higher pain levels and poorer quality of life after surgery compared with patients without that (38). In view of the significantly increased risk of $\mathrm{POH}$, we believe that it may be a reasonable strategy to take appropriate prophylactic drugs early to prevent the development of $\mathrm{POH}$ in patients with a history of headache (32).

The other four independent risk factors for $\mathrm{POH}$ identified in this study have never been reported before, including LVEF, WBC count, albumin, and CPB time. Low LVEF often means poor cardiac function, which may lead to decreased blood pressure and headache. Paraskevi and colleagues found that intraoperative hypotension was independently associated with $\mathrm{POH}$ (13). Improving cardiac function before surgery may help to reduce $\mathrm{POH}$. The elevation of WBC count was significantly associated 
with the development of $\mathrm{POH}$ in the preoperative model, which may have relationship with the acute phase systemic inflammatory response. Furthermore, a genetic correlation between migraine headache and white blood cell count was found by Katherine and colleagues, also indicating a close association (36). Low preoperative albumin was also identified as an independent predictor in the preoperative model. Commonly, low serum albumin level is known as an indicator of poor nutritional state which also means the low levels of other nutrients. A recent review summarized that the deficiency of various nutrients was associated with migraine headache (39), which can partly account for our results. Therefore, improving preoperative nutritional status, including the use of some nutraceuticals, may play an important role in $\mathrm{POH}$ prevention.

Not surprisingly, the duration of $\mathrm{CPB}$ was independently associated with the development of $\mathrm{POH}$ in patients undergoing HVS. Longer duration of CPB always means longer duration of surgery, which is associated with a prolonged forced position and larger doses of anaesthesia drugs. Maria and colleagues reported that the duration of surgery longer than four hours was associated with 3.7 -fold increased odds of $\mathrm{POH}$ (16). The use of anaesthesia drugs was also reported to be associated with the development of $\mathrm{POH}$ (13). In addition, the $\mathrm{CPB}$ itself can cause brain damage and $\mathrm{POH}$ through several ways, which mainly include cerebral hypoxia, edema, hemorrhage, and embolism (40-42). Taking appropriate strategies to better protect the brain during CPB may be effective in reducing POH (42).

Several other factors have been reported to be associated with the development of $\mathrm{POH}$ after surgery in other diseases but were not identified as significant predictors in our analysis, including age $(10,16,20,37,43)$, anaesthesia drugs (13), depression and anxiety disorders $(9,44)$, and caffeine withdrawal (45). The big difference of the risk factors for $\mathrm{POH}$ in patients undergoing HVS and surgery for other diseases further emphasized the necessity of this study.

Our nomogram models may play an important role in predicting individualized risk and may have clinical utility in the reduction of $\mathrm{POH}$ after HVS. Appropriate preventative measures and specific interventions targeting high-risk populations identified by the nomogram models may produce great clinical and economic benefits.

There were several limitations in this study. First of all, this was a single-center study. Although the full prediction model was well validated in the independent validation set, the absence of external validation in the dataset of other centers may limit its generalizability. Second, some potential predictors that may relate to the occurrence of $\mathrm{POH}$ were not included in our analysis, such as anxiety disorders and caffeine withdrawal. Third, the duration of this study was relatively short and limited to the hospitalization period. Long-term follow-up after discharge was not performed, which may underestimate the true incidence of $\mathrm{POH}$ after HVS. Fourth, we included $\mathrm{POH}$ as the primary endpoint, but the type and severity of $\mathrm{POH}$ were not evaluated in this study. Multicenter studies with larger sample sizes are required to overcome these weaknesses and to better study the predictors of $\mathrm{POH}$.

\section{Conclusions}

$\mathrm{POH}$ was prevalent in patients undergoing HVS. To our knowledge, this is the first study to identify predictors and develop multivariate prediction models for $\mathrm{POH}$ in patients undergoing HVS. In this study, we developed and validated a full nomogram model using preoperative and intraoperative predictors, and a preoperative model using only preoperative predictors. Both of the two nomogram models performed well in terms of discrimination and calibration. The decision and clinical impact curves also indicated remarkable predictive power and good clinical utility. Through individualized risk assessment and identification of high-risk patients, the nomogram models can help inform clinician-patient decision-making. The potential clinical applications lie in risk modification and targeted interventions to reduce the incidence of $\mathrm{POH}$ in patients undergoing HVS.

\section{Acknowledgments}

We would like to thank $D r$. Fobn $S$ for his help in polishing our paper.

Funding: This work was supported by the National Natural Science Foundation of China (Grant No. 81800413).

\section{Footnote}

Reporting Checklist: The authors have completed the TRIPOD reporting checklist. Available at https://dx.doi. org/10.21037/jtd-21-644

Data Sharing Statement: Available at https://dx.doi. org/10.21037/jtd-21-644

Peer Review File: Available at https://dx.doi.org/10.21037/ jtd-21-644 
Conflicts of Interest: All authors have completed the ICMJE uniform disclosure form (available at https://dx.doi. org/10.21037/jtd-21-644). The authors have no conflicts of interest to declare.

Etbical Statement: The authors are accountable for all aspects of the work in ensuring that questions related to the accuracy or integrity of any part of the work are appropriately investigated and resolved. The study was conducted according to the Declaration of Helsinki (as revised in 2013). The Ethics Committee of Tongji Medical College of Huazhong University of Science and Technology (IORG No. IORG0003571) approved this study. The need for informed consent was waived because of the retrospective study design.

Open Access Statement: This is an Open Access article distributed in accordance with the Creative Commons Attribution-NonCommercial-NoDerivs 4.0 International License (CC BY-NC-ND 4.0), which permits the noncommercial replication and distribution of the article with the strict proviso that no changes or edits are made and the original work is properly cited (including links to both the formal publication through the relevant DOI and the license). See: https://creativecommons.org/licenses/by-nc-nd/4.0/.

\section{References}

1. Roth GA, Mensah GA, Johnson CO, et al. Global Burden of Cardiovascular Diseases and Risk Factors, 1990-2019: Update From the GBD 2019 Study. J Am Coll Cardiol 2020;76:2982-3021.

2. GBD 2019 Diseases and Injuries Collaborators. Global burden of 369 diseases and injuries in 204 countries and territories, 1990-2019: a systematic analysis for the Global Burden of Disease Study 2019. Lancet 2020;396:1204-22.

3. Otto CM, Nishimura RA, Bonow RO, et al. 2020 ACC/ AHA Guideline for the Management of Patients With Valvular Heart Disease: A Report of the American College of Cardiology/American Heart Association Joint Committee on Clinical Practice Guidelines. Circulation 2021;143:e72-e227.

4. Pogoda L, Nijdam JS, Smeeing DPJ, et al. Postoperative headache after surgical treatment of cerebellopontine angle tumors: a systematic review. Eur Arch Oto-Rhino-L 2021. [Epub ahead of print]. doi: 10.1007/s00405-02106627-6.

5. Jang MK, Oh EG, Lee H, et al. Postoperative Symptoms and Quality of Life in Pituitary Macroadenomas Patients. J Neurosci Nurs 2020;52:30-6.

6. Jang MK, Park CG, Jang S, et al. Prevalence and Impact of Postoperative Headaches in Nonfunctioning Pituitary Macroadenoma Patients: A Longitudinal Cohort Study. World Neurosurg 2020;133:e633-9.

7. Santa Maria C, Santa Maria PL, Bulsara V, et al. Longterm quality of life in patients with vestibular schwannoma managed with microsurgery. J Laryngol Otol 2019;:1-7.

8. Sabab A, Sandhu J, Bacchi S, et al. Postoperative headache following treatment of vestibular schwannoma: A literature review. J Clin Neurosci 2018;52:26-31.

9. Rocha-Filho PA. Post-craniotomy headache: a clinical view with a focus on the persistent form. Headache 2015;55:733-8.

10. Ryzenman JM, Pensak ML, Tew JM Jr, et al. Headache: a quality of life analysis in a cohort of 1,657 patients undergoing acoustic neuroma surgery, results from the acoustic neuroma association. Laryngoscope 2005;115:703-11.

11. Su H, Li B, Wang J, et al. Headache attributed to cranial venous sinus stenting: A case series and literature review. Cephalalgia 2019;39:1277-83.

12. Guenther F, Swozil F, Heber S, et al. Pre- and postoperative headache in patients with meningioma. Cephalalgia 2019;39:533-43.

13. Matsota PK, Christodoulopoulou TC, Batistaki CZ, et al. Factors associated with the presence of postoperative headache in elective surgery patients: a prospective single center cohort study. J Anesth 2017;31:225-36.

14. Kisser U, Adderson-Kisser C, Förster M, et al. Prevalence of Headache in Cochlear Implant Patients: A Crosssectional Study. Otol Neurotol 2016;37:1555-9.

15. Molnár L, Simon É, Nemes R, et al. Postcraniotomy headache. J Anesth 2014;28:102-11.

16. de Oliveira Ribeiro Mdo C, Pereira CU, Sallum AM, et al. Immediate post-craniotomy headache. Cephalalgia 2013;33:897-905.

17. Rocha-Filho PA, Gherpelli JL, de Siqueira JT, et al. Post-craniotomy headache: a proposed revision of IHS diagnostic criteria. Cephalalgia 2010;30:560-6.

18. Bitargil M, El Kılıç H, et al. Our experience regarding patients with headache, vomiting, and urinary retention following endothermal ablation of the greater saphenous vein under spinal anesthesia: Gender type, age interval, and procedural risk factors are important. Vascular 2020;28:591-6.

19. Yilmaz G, Akca A, Kiyak H, et al. Elevation in optic nerve sheath diameter due to the pneumoperitoneum 
and Trendelenburg is associated to postoperative nausea, vomiting and headache in patients undergoing laparoscopic hysterectomy. Minerva Anestesiol 2020;86:270-6.

20. Liang B, Shetty SR, Omay SB, et al. Predictors and incidence of orthostatic headache associated with lumbar drain placement following endoscopic endonasal skull base surgery. Acta Neurochir (Wien) 2017;159:1379-85.

21. Gill PS, Guest C, Rabey PG, et al. Perioperative headache and day case surgery. Eur J Anaesthesiol 2003;20:401-3.

22. Platzbecker K, Zhang MB, Kurth T, et al. The association between migraine and hospital readmission due to pain after surgery: A hospital registry study. Cephalalgia 2019;39:286-95.

23. Wang EHZ, Sunderland S, Edwards NY, et al. A Single Prophylactic Dose of Ondansetron Given at Cessation of Postoperative Propofol Sedation Decreases Postoperative Nausea and Vomiting in Cardiac Surgery Patients: A Randomized Controlled Trial. Anesth Analg 2020;131:1164-72.

24. DeLong ER, DeLong DM, Clarke-Pearson DL, et al. Comparing the areas under two or more correlated receiver operating characteristic curves: a nonparametric approach. Biometrics 1988;44:837-45.

25. Vickers AJ, Elkin EB. Decision curve analysis: a novel method for evaluating prediction models. Med Decis Making 2006;26:565-74.

26. Takenaka S, Makino T, Sakai Y, et al. Prognostic impact of intra- and postoperative management of dural tear on postoperative complications in primary degenerative lumbar diseases. Bone Joint J 2019;101-B:1115-21.

27. Myles PS, Hunt JO, Moloney JT, et al. Postoperative 'minor' complications. Comparison between men and women. Anaesthesia 1997;52:300-6.

28. Hipolito Rodrigues MA, Maitrot-Mantelet L, PluBureau G, et al. Migraine, hormones and the menopausal transition. Climacteric 2018;21:256-66.

29. Gupta S, McCarson KE, Welch KM, et al. Mechanisms of pain modulation by sex hormones in migraine. Headache 2011;51:905-22.

30. MacGregor EA. Oestrogen and attacks of migraine with and without aura. Lancet Neurol 2004;3:354-61.

31. Rist PM, Winter AC, Buring JE, et al. Migraine and the risk of incident hypertension among women. Cephalalgia 2018;38:1817-24.

32. Evers $S$. Treatment of migraine with prophylactic drugs. Expert Opin Pharmacother 2008;9:2565-73.

33. Diener HC, Küper M, Kurth T, et al. Migraine-associated risks and comorbidity. J Neurol 2008;255:1290-301.

34. Charles A. The pathophysiology of migraine: implications for clinical management. Lancet Neurol 2018;17:174-82.

35. Ripa P, Ornello R, Pistoia F, et al. The renin-angiotensin system: a possible contributor to migraine pathogenesis and prophylaxis. Expert Rev Neurother 2014;14:1043-55.

36. Siewert KM, Klarin D, Damrauer SM, et al. Cross-trait analyses with migraine reveal widespread pleiotropy and suggest a vascular component to migraine headache. Int J Epidemiol 2020;49:1022-31.

37. Valentinis L, Tuniz F, Valent F, et al. Headache attributed to intracranial tumours: a prospective cohort study. Cephalalgia 2010;30:389-98.

38. Yabuki S, Takatsuki K, Otani K, et al. Headache in Patients with Cervical Spondylotic Myelopathy. Pain Res Manag 2020;2020:8856088.

39. Nattagh-Eshtivani E, Sani MA, Dahri M, et al. The role of nutrients in the pathogenesis and treatment of migraine headaches: Review. Biomed Pharmacother 2018;102:317-25.

40. Zhu S, Sai X, Lin J, et al. Mechanisms of perioperative brain damage in children with congenital heart disease. Biomed Pharmacother 2020;132:110957.

41. Caldas JR, Haunton VJ, Panerai RB, et al. Cerebral autoregulation in cardiopulmonary bypass surgery: a systematic review. Interact Cardiovasc Thorac Surg 2018;26:494-503.

42. Salameh A, Dhein S, Dähnert I, et al. Neuroprotective Strategies during Cardiac Surgery with Cardiopulmonary Bypass. Int J Mol Sci 2016;17:1945.

43. Bowers CA, Gurgel RK, Brimley C, et al. Surgical Treatment of Vestibular Schwannoma: Does Age Matter? World Neurosurg 2016;96:58-65.

44. Magalhães JE, Azevedo-Filho HR, Rocha-Filho PA, et al. The risk of headache attributed to surgical treatment of intracranial aneurysms: a cohort study. Headache 2013;53:1613-23.

45. Hampl KF, Schneider MC, Rüttimann U, et al. Perioperative administration of caffeine tablets for prevention of postoperative headaches. Can J Anaesth 1995;42:789-92.

Cite this article as: Wang D, Huang X, Wang H, Le S, Du X. Predictors and nomogram models for postoperative headache in patients undergoing heart valve surgery. J Thorac Dis 2021;13(7):4236-4249. doi: 10.21037/jtd-21-644 